\title{
Conocimiento y prácticas de prevención de cáncer de cuello uterino en mujeres con lesiones histopatológicas. Cuenca, Ecuador 2021
}

\author{
Knowledge and practices of cervical cancer prevention among women with histopathological \\ lesions. Cuenca, Ecuador 2021
}

\author{
José Ortiz-Segarra ${ }^{*^{*}}$ iD, Bernardo Vega ${ }^{2}$ iD, Vivian Alejandra Neira ${ }^{2}$ iD, Lorena Mora-Bravo ${ }^{1}$ (D), \\ Gabriela Guerra-Astudillo $^{1}{ }^{\text {iD }}$, José Ortíz-Mejía ${ }^{1}$ (D), Verónica Pérez-Paredes ${ }^{1}$ iD \\ ${ }^{1}$ Facultad de Ciencias Médicas, Universidad de Cuenca, Av. Pje. del Paraíso, Cuenca, Ecuador. \\ ${ }^{2}$ Facultad de Ciencias Químicas, Universidad de Cuenca, Av. 12 de abril, Cuenca, Ecuador. \\ *Autor de correspondencia: jose.ortiz@ucuenca.edu.ec \\ Fecha de recepción: 9 de julio de 2021 - Fecha de aceptación: 31 de agosto de 2021
}

\begin{abstract}
RESUMEN
Los conocimientos y las prácticas son esenciales para promover la salud, prevenir enfermedades, mantener la salud y hacer frente a las enfermedades en general y en particular al cáncer cérvico-uterino. El objetivo del presente estudio fue describir las características sociodemográficas y prácticas de prevención en mujeres con lesiones histopatológicas de cuello uterino, para lo cual se entrevistó a 174 mujeres de entre 30 a 89 años de edad, atendidas en los hospitales de SOLCA y Vicente Corral de la ciudad de Cuenca. Los resultados muestran que las participantes en su mayoría residentes en zonas urbana y urbanomarginal, casadas, con educación primaria y secundaria, con dificultades económicas y auto identificación mestiza, presentaron con mayor frecuencia lesiones histopatológicas tipo LIEAG, LIEBG y Cáncer. Si bien poseen información sobre el VPH, muy pocas conocen que es la principal causa del CCU, que se transmite sexualmente, que existen vacunas y pruebas de detección temprana. Entre los factores facilitadores para la realización del examen de Papanicolaou (PAP) están: la visita de seguimiento, recomendación médica, iniciativa propia y recomendación de una amiga o familiar; en tanto que los factores personales, problemas en el servicio de salud, dificultades económicas y familiares, constituyeron las principales barreras.
\end{abstract}

Palabras clave: Conocimiento y prácticas de prevención, cáncer de cuello uterino, virus del papiloma humano.

\begin{abstract}
Knowledge and practices are essential to promote health, prevent disease, maintain health, and cope with diseases in general and cervical cancer in particular. The objective of this study was to describe the sociodemographic characteristics and prevention practices in women with histopathological lesions of the cervix, for which 174 female patients, between 30 and 89 years of age, were interviewed at the SOLCA and Vicente Corral hospitals in the city of Cuenca. The results show that the participants, mostly living in urban and marginal urban areas, married, with primary and secondary education, with economic difficulties and mestizo self-identification, more frequently presented histopathological lesions of the LIEAG, LIEBG and Cancer type. Although they have information about HPV, very few know that it is the main cause of CCU, that it is transmitted sexually, that there are vaccines and early detection tests. Among the facilitating factors to carry out the Pap smear are: the follow-up visit, medical recommendation, own initiative and recommendation of a friend or relative; while personal factors, problems in the health service, economic and family difficulties, constitute the main barriers.
\end{abstract}

Keywords: Knowledge and prevention practices, cervical cancer, human papilloma virus.

\section{INTRODUCCIÓN}

La A nivel global, el cáncer de cuello uterino (CCU) es el cuarto cáncer más frecuente en población femenina. En el año 2020 fueron diagnosticados 604,127 nuevos casos, lo que representa el $6.5 \%$ del total de muertes por cáncer (WHO \& IARC, 2020a). De las 311,000 defunciones que ocurren anualmente por CCU, cerca del $85 \%$ se producen en los países en vías de desarrollo, a pesar de ser una enfermedad prevenible (Stelzle et al., 2021). Esta patología se presenta en forma desigual en los países desarrollados y en vías de desarrollo, de igual manera entre las regiones de un mismo país. En el año 2012 la mortalidad por CCU representó el 7.5\% del total de la mortalidad por cáncer a nivel mundial, con una tasa de 6.8 por 100,000 mujeres, en España es de 2.1 y en Estados 
Unidos 2.8; en tanto que en los países pobres de Asia, África, América Latina y El Caribe se produce el $87 \%$ de los fallecimientos por CCU (Amengual et al., 2020).

En mujeres con afectación del sistema inmunitario la aparición del CCU puede tardar entre 5 a 10 años; mientras que, en aquellas con un sistema inmunitario normal tarda entre 15 a 20 años. La alta tasa de mortalidad mundial por este problema (tasa estandarizada por edad: 6,9 por 100,000 en 2018) podría reducirse con intervenciones eficaces como la detección temprana de alteraciones en citología cervical, a través del examen de Papanicolaou y con la inmunización contra el virus del papiloma humano (VPH) (WHO, 2021). En Ecuador en el año 2020 se registraron 1,534 nuevos casos de CCU, representando la segunda causa de neoplasia maligna en mujeres, luego del cáncer de mama y la mortalidad por esta causa ocupó el tercer lugar después del cáncer de estómago y de mama (WHO \& IARC, 2020b).

La Agencia Internacional de Investigación de cáncer (IARC, por sus siglas en inglés) reconoce que la infección por los VPH de alto riesgo constituye una causa necesaria para el desarrollo de CCU; no obstante, se admite también que la sola infección por VPH no es suficiente puesto que la mayoría de las infecciones son asintomáticas y desaparecen en un periodo de 2 años (IARC, 2007). Si bien se han caracterizado más de 200 genotipos de HPV, dos (HPV 16 y 18) representan el $80 \%$ del cáncer cervical inducido por HPV en todo el mundo, y otros doce $(31,33$, $35,39,45,51,52,56,58,59,66$ y 68$)$ también son potenciales conductores de alto riesgo de cáncer cervical (Landy et al., 2016; Schmitt et al., 2013; Lorenzi et al., 2015). Estos 14 genotipos de VPH se consideran de alto riesgo para el desarrollo del cáncer cervical. Por lo tanto, la detección estándar de VPH se basa en la discriminación entre estos 14 genotipos de alto riesgo y otros genotipos de bajo riesgo. Sin embargo, hasta el $40 \%$ de las infecciones por VPH son de genotipo mixto. En consecuencia, la identificación del genotipo presente en cada infección por VPH es importante para determinar el riesgo general de una mujer de desarrollar CCU (Lorenzi et al., 2015). En varios estudios realizados en España (Sánchez, 2012), China (Mijit et al., 2015), Estados Unidos (Krishnan \& Thomas, 2016), entre otros, se ha demostrado que la infección por genotipos de alto riesgo está presente en casi todos los casos de CCU, principalmente por los genotipos 16 y 18 .

La evidencia científica existente respalda que los factores de riesgo tales como la edad (Torres-Poveda et al., 2019), bajo nivel socioeconómico y educativo (Karadag Arli et al., 2019), el inicio temprano de la vida sexual (Muñoz et al., 2003), un mayor número de parejas sexuales (TorresPoveda et al., 2019; Liu et al., 2015), infecciones de transmisión sexual (Kashyap et al., 2019), un mayor número de partos (Karadag Arli et al., 2019; Muñoz et al., 2002), uso de anticonceptivos orales por más de cuatro años (Torres-Poveda et al., 2019), el tabaquismo activo (Torres-Poveda et al., 2019; Sugawara et al., 2019) у pasivo (Su et al., 2018), aumentan de manera convincente el riesgo de cáncer de cuello uterino, de ahí la importancia de este estudio mediante el cual se contribuirá en el

\footnotetext{
${ }^{1}$ ELEVATE es el acrónimo de un proyecto financiado por la Unión Europea con el objetivo de establecer un equipo multidisciplinario compuesto por fabricantes y expertos de Europa y de la Comunidad de Estados de América Latina y el Caribe, para mejorar la adecuación y cobertura global de las pruebas del cáncer de cuello uterino, en particular para
}

esclarecimiento de los conocimientos sobre el CCU y las prácticas que realizan las mujeres para su prevención, así como las barreras y facilitadores para la realización del PAP.

\section{POBLACIÓN Y MÉTODO}

Este artículo forma parte del proyecto EarLy dEtection of cerVical cAncer in hard-to-reach populations of women through portable and pointof-care HPV TEsting (ELEVATE ${ }^{1}$ ) que cuenta con financiamiento de la Unión Europea, cuyo propósito es identificar a las mujeres de difícil acceso en Bélgica, Brasil, Ecuador y Portugal, abordar las barreras de acceso y diseñar estrategias para la detección genómica y proteómica del VPH en el lugar de la atención.

Se trata de un estudio descriptivo de corte transversal, con base en el análisis de 174 entrevistas realizadas a mujeres de 30 años y más de edad que acudieron de manera espontánea a la consulta en el Instituto del Cáncer SOLCA de la ciudad de Cuenca y Hospital Vicente Corral, durante el período octubre de 2019 a junio de 2021. Considerando que únicamente disponíamos de la información de mujeres con lesiones histopatológicas de cuello uterino, sin tener la misma información de mujeres sin lesiones, no fue posible analizar la asociación entre la infección por VPH con la salud, las variables de comportamiento y el perfil sociodemográfico; en este marco realizamos el análisis descriptivo que consta en los resultados.

Los datos fueron recolectados por el equipo de investigación entrenado, mediante un formulario estructurado. Las mujeres fueron abordadas en sala de espera, y quienes manifestaron su voluntad de participar en el estudio fueron conducidas a una oficina privada donde se realizaron las entrevistas, por un tiempo promedio de 10 minutos, previa firma del consentimiento informado; luego, las mujeres entrevistadas fueron acompañadas al consultorio de ginecología donde el especialista ginecólogo le realizó el interrogatorio, examen físico y la toma de muestras para el examen de Papanicolaou, de acuerdo con el protocolo establecido por la institución.

El formulario contiene preguntas relacionadas con las variables sociodemográficas, factores de riesgo, conocimientos y prácticas de prevención del cáncer de cuello uterino. El protocolo del estudio ELEVATE fue aprobado por el Comité de Bioética en Investigación del Área de la Salud de la Universidad de Cuenca (código 2018-074EO-I). La información recolectada fue transcrita a una base de datos en el programa Excel versión 2016; a cada registro se le asignó un código numérico de 21 dígitos en el que se detalla el país de origen (1), institución donde se atendió (1), fecha de nacimiento (8), número de paciente (3) y fecha de la toma de la muestra (8), como mecanismo de anonimización. La base de datos fue protegida con una clave y tenían acceso únicamente los investigadores. Las variables cualitativas fueron

poblaciones específicas de mujeres que, al no someterse a exámenes de detección con regularidad (poblaciones de difícil acceso), tienen un mayor riesgo de desarrollar cáncer de cuello uterino. 
analizadas mediante frecuencias y porcentajes y de las cuantitativas se obtuvo promedios, valor máximo, mínimo y desvío estándar.

\section{RESULTADOS}

Como se muestra en la Tabla 1, la edad promedio de las participantes fue de 48 años, con una edad máxima de 89 , una mínima de 30 y una desviación estándar de 12.73; cerca de la tercera parte de las mujeres entrevistadas en el Instituto del Cáncer SOLCA de la ciudad de Cuenca y en el Hospital Vicente Corral se ubican el grupo de entre 30 a 40 años de edad; en similar proporción se encuentra el grupo de participantes de 41 a 50 años y algo más de la tercera parte se ubican en el grupo eterio de 51 años y más. La mitad de las participantes reside en la zona urbana, cerca de la tercera parte en zona rural y el resto habita en barrios urbano marginales. Siete de cada diez proceden de la provincia del Azuay; una cuarta parte provienen de las provincias de Cañar, El Oro y Morona Santiago y en menor proporción de otras provincias del país. En lo referente al medio de transporte que utilizan para llegar a un centro de salud para realizarse el examen de PAP, más de la mitad llega a pie, una de cada tres utiliza el transporte público, una de cada cinco dispone de vehículo propio y solamente una mujer acude utilizando motocicleta. Con respecto al nivel educativo, cuatro de cada diez participantes han alcanzado la primaria, una de cada cuatro completó los estudios secundarios, una de cada cinco realizó estudios universitarios, cerca de una de cada diez se educó en centros de alfabetización y el 6, 25\% de las mujeres son analfabetas. En cuanto al estado civil, más de la mitad de las mujeres mencionaron estar casadas, una de cada ocho manifestó ser soltera o divorciada, y en menor porcentaje indicaron ser viudas. Nueve de cada diez entrevistadas se auto identificó como mestiza, las demás se identificaron como blancas, indígenas, afroecuatorianas y morenas. En lo referente a la situación económica, seis de cada diez participantes han manifestado que tienen problemas para pagar las facturas de sus gastos personales y familiares; así mismo, más de la mitad han expresado dificultades para solventar los gastos relacionados con la alimentación.

Considerando que en el proyecto ELEVATE antes mencionado, está orientado a seleccionar mujeres tanto con lesiones histopatológicas, como aquellas sin lesiones histopatológicas del cuello uterino, con la finalidad de diseñar e implementar un prototipo capaz de detectar, tanto los 14 genotipos $(16,18,31,33,35,39,45,51,52$, $56,58,59,66$ y 68 ) del virus del VPH, como la detección

Tabla 1. Características demográficas y socioeconómicas.

\begin{tabular}{|c|c|c|c|}
\hline Parámetro & Especificación & $\mathrm{N}^{\circ}$ & $\%$ \\
\hline \multirow[t]{3}{*}{ Edad } & 30 a 40 & 53 & 30.64 \\
\hline & 41 a 50 & 53 & 30.64 \\
\hline & 51 y más & 67 & 38.73 \\
\hline \multirow[t]{3}{*}{ Residencia } & Urbano & 86 & 49.71 \\
\hline & Urbano marginal & 36 & 20.81 \\
\hline & Rural & 51 & 29.48 \\
\hline \multirow{4}{*}{$\begin{array}{l}\text { Llegada a centro de } \\
\text { salud }\end{array}$} & A pie & 87 & 50.29 \\
\hline & Vehículo propio & 28 & 16.18 \\
\hline & Transporte público & 57 & 32.95 \\
\hline & Motocicleta & 1 & 0.58 \\
\hline \multirow{5}{*}{$\begin{array}{l}\text { Nivel más alto de } \\
\text { educación }\end{array}$} & Sin educación & 15 & 9.09 \\
\hline & Centro de & 17 & 10.30 \\
\hline & Educación primaria & 57 & 34.55 \\
\hline & Educación secundaria & 45 & 27.27 \\
\hline & Universidad & 31 & 18.79 \\
\hline \multirow[t]{5}{*}{ Estado civil } & Soltera & 32 & 19.39 \\
\hline & Unión Libre & 19 & 11.52 \\
\hline & Casada & 79 & 47.88 \\
\hline & Divorciada o separada & 17 & 10.30 \\
\hline & Viuda & 18 & 10.91 \\
\hline \multirow{4}{*}{$\begin{array}{l}\text { Problemas para } \\
\text { pagar las facturas }\end{array}$} & Nunca & 58 & 35.15 \\
\hline & A veces & 68 & 41.21 \\
\hline & A menudo & 19 & 11.52 \\
\hline & Siempre & 20 & 12.12 \\
\hline \multirow{4}{*}{$\begin{array}{l}\text { Problemas para } \\
\text { pagar la comida }\end{array}$} & Nunca & 66 & 40.00 \\
\hline & A veces & 64 & 38.79 \\
\hline & A menudo & 20 & 12.12 \\
\hline & Siempre & 15 & 9.09 \\
\hline \multirow{5}{*}{$\begin{array}{l}\text { Autoidentificación } \\
\text { étnica }\end{array}$} & Blanca & 5 & 3.03 \\
\hline & Mestiza & 154 & 93.33 \\
\hline & Indígena & 5 & 3.03 \\
\hline & Afroecuatoriana & 0 & 0.00 \\
\hline & Morena & 1 & 0.61 \\
\hline
\end{tabular}


simultánea de biomarcadores p16 (marcador de antiproliferación) y Ki67 (marcador de proliferación), para la identificación y confirmación de verdaderos positivos para CCU en la citología vaginal, en la Tabla 2 podemos apreciar los resultados histopatológicos clasificados según el sistema Bethesda, en orden de frecuencia: en primer lugar, un grupo que representa cerca de la tercera parte, sin lesiones histopatológicas (Normal); en segundo y tercer lugar, están con algo más de la quinta parte, los grupos con Lesión Escamosa Intraepitelial de Alto Grado (LIEAG) y Lesión Escamosa Intraepitelial de Bajo Grado (LIEBG); el cuarto lugar ocupa el grupo con diagnóstico de cáncer, que está presente en una de cada siete mujeres; en una de cada once mujeres se identificó Atipia de Células Escamosas de Significado Indeterminado (ASCUS, por sus siglas en inglés) y en menor porcentaje se encontró células escamosas atípicas en las cuales los cambios son sugestivos de una Lesión Intraepitelial Escamosa de Alto Grado (ASC H) y en último lugar está el grupo con Células Glandulares Atípicas (ACG). En las mujeres con cualquier tipo de lesiones se pretende averiguar los conocimientos que poseen sobre el VPH y el CCU, así como las prácticas que realizan para la detección temprana del CCU.

Tabla 2. Tipo de lesión según sistema Bethesda.

\begin{tabular}{lrr}
\hline Tipo de lesión & $\mathrm{N}^{\mathrm{o}}$ & $\%$ \\
\hline Sin lesión & 80 & 31.50 \\
LIEAG & 55 & 21.65 \\
LIEBG & 50 & 19.69 \\
Cáncer & 36 & 14.57 \\
ASCUS & 22 & 8.66 \\
ASC H & 7 & 2.76 \\
ACG & 3 & 1.18 \\
\hline Fuente: informe de resultados histopatológicos
\end{tabular}

En relación a los conocimientos sobre el VPH (Tabla 3), cuatro de cada diez entrevistadas manifestaron que nunca han escuchado nada al respecto. De entre las mujeres que sí han escuchado sobre el VPH, algo más de la cuarta parte se enteraron a través del médico que les atendió, en menor proporción indican que se enteraron a través de amigos o familiares, la radio o televisión, el sistema educativo (escuela y colegio) y a través del Internet. Cuando se les preguntó si el VPH causa CCU, una de cada seis indicó que sí conocía al respecto, cerca de la mitad dijeron que sabían que el virus del papiloma humano se puede transmitir sexualmente. Acerca de la vacuna y las pruebas para la detección del VPH, la mayoría de las entrevistadas contestaron que no escucharon nada al respecto.

Según los datos expuestos en la Tabla 4 la mayoría de las entrevistadas manifestaron que acuden al establecimiento de salud para una visita de seguimiento, solamente una de cada seis asistía al establecimiento de salud para realizarse la prueba de PAP, las demás fueron al establecimiento de salud por invitación para participar en el estudio. Al preguntarles sobre las razones por las que se realiza el PAP, solamente cuatro de cada diez indicaron que lo hacían por iniciativa propia, una proporción similar de las mujeres indicaron que fue porque el médico les recomendó ya sea porque tenía o no molestias; en menor proporción por recomendaciones de una amiga o familiar. Con respecto a la frecuencia de los exámenes, una de cada ocho mujeres indicó que nunca se había realizado un PAP y tres de cada cuatro se realizaron este examen más de una vez. Una de cada cinco participantes indicó que se ha realizado el PAP hace más de cinco años. Cerca de la mitad de las mujeres señalaron que el primer examen se realizó cuando tenía entre 20 a 30 años de edad y en proporción similar cuando tuvieron más de 30 años. Entre las principales razones por las que no se realizaron el PAP, en orden de frecuencia, indican: porque no tenía tiempo, no confía en los doctores, no había oído hablar del cáncer cervical, miedo, no pensó que estaba en riesgo y no pensó que fuera importante; las demás causas están relacionadas principalmente con el servicio de salud, aspectos personales y los relacionados con la pareja que no estuvo de acuerdo que se realice el examen.

Tabla 3. Conocimientos sobre el VPH y el CCU en mujeres con lesiones histopatológicas del cuello uterino.

\begin{tabular}{lrr}
\hline Escuchó sobre el virus del papiloma humano & No. & $\%$ \\
\hline No & 68 & 41.21 \\
Sí, me enteré a través del médico & 42 & 25.45 \\
Sí, me enteré a través de amigos o familiares & 22 & 13.33 \\
Sí, lo escuché en la radio o en la televisión & 23 & 13.94 \\
Sí, me enteré en el sistema educativo & 9 & 5.45 \\
A través del Internet & 1 & 0.61 \\
\hline Sabía que el virus del papiloma humano causa cáncer & & \\
\hline Sí & 27 & 16.36 \\
No & 73 & 44.24 \\
No responde & 65 & 39.39 \\
\hline Sabía que el virus del papiloma humano se puede transmitir & \\
\hline No & 23 & 13.94 \\
Sí & 76 & 46.06 \\
No responde & 66 & 40.00 \\
\hline Escuchó sobre la vacuna para VPH & \\
\hline No & 109 & 66.06 \\
Sí & 56 & 33.94 \\
\hline Escuchó sobre las pruebas de detección del VPH & \\
\hline No & 122 & 73.94 \\
Sí & 43 & 26.06 \\
\hline Fuente: entrevistas
\end{tabular}


Tabla 4. Prácticas de prevención para el CCU en mujeres con lesiones histopatológicas del cuello uterino.

\begin{tabular}{|c|c|c|}
\hline Razón de visita al establecimiento de salud & No & $\%$ \\
\hline Visita de seguimiento & 117 & 70.91 \\
\hline Para la prueba de detección de cáncer cervical & 30 & 18.18 \\
\hline Me invitaron al estudio & 18 & 10.91 \\
\hline \multicolumn{3}{|l|}{ Razón por la que se realiza el PAP } \\
\hline Por iniciativa propia & 67 & 40.61 \\
\hline Por recomendaciones de un amigo/a o familiar & 18 & 10.91 \\
\hline Mi médico lo recomendó sin molestias & 35 & 21.21 \\
\hline Mi médico lo recomendó porque tenía molestias & 42 & 25.45 \\
\hline No responde & 3 & 1.82 \\
\hline \multicolumn{3}{|l|}{ Número de veces que ha sido examinada } \\
\hline Nunca & 20 & 12.12 \\
\hline Una vez & 18 & 10.91 \\
\hline Más de una vez & 127 & 76.97 \\
\hline \multicolumn{3}{|l|}{ Última vez que fue examinada } \\
\hline Hace 1 año & 64 & 38.79 \\
\hline Hace más de un año y menos de 5 & 42 & 25.45 \\
\hline Hace más de 5 años & 35 & 21.21 \\
\hline No responde & 20 & 12.12 \\
\hline No recuerda & 4 & 2.42 \\
\hline \multicolumn{3}{|l|}{ Tiempo del último examen } \\
\hline Hace 1 año & 58 & 35.15 \\
\hline Hace más de un año y menos de 5 & 29 & 17.58 \\
\hline Hace más de 5 años & 19 & 11.52 \\
\hline No corresponde & 38 & 23.03 \\
\hline No recuerda & 21 & 12.73 \\
\hline \multicolumn{3}{|l|}{ Edad del primer examen } \\
\hline Menor de 20 años & 9 & 5.45 \\
\hline Entre 20 a 30 años & 81 & 49.09 \\
\hline Más de 30 años & 75 & 45.45 \\
\hline \multicolumn{3}{|l|}{ Razón por la que no se realizó el PAP (varias opciones) } \\
\hline No tuve tiempo & 27 & 16.36 \\
\hline No pensé que fuera importante & 7 & 4.24 \\
\hline No había oído hablar de cáncer cervical & 22 & 13.33 \\
\hline Descuido & 9 & 5.45 \\
\hline No pensé que estaba en riesgo & 8 & 4.85 \\
\hline No confío en los doctores & 25 & 15.15 \\
\hline No tenía dinero & 7 & 4.24 \\
\hline Miedo & 14 & 8.48 \\
\hline No tenía molestias & 4 & 2.42 \\
\hline Vergüenza & 1 & 0.61 \\
\hline Pérdida de exámenes por el personal de salud & 6 & 3.64 \\
\hline Burocracia & 5 & 3.03 \\
\hline Incomodidad & 1 & 0.61 \\
\hline Le decían que no debe hacerse & 1 & 0.61 \\
\hline Porque estaba embarazada & 1 & 0.61 \\
\hline
\end{tabular}

\section{DISCUSIÓN}

En el presente estudio las mujeres que acudieron espontáneamente a la consulta del área de Ginecología del Instituto de Cáncer SOLCA de la ciudad de Cuenca y del Hospital Vicente Corral, tienen un promedio de edad de 48 años, son residentes en las zonas urbana, rural y urbano-marginal, y cerca de la mitad casadas. En su mayoría son mujeres con educación entre primaria y secundaria, con dificultades económicas para solventar sus necesidades básicas y con auto identificación étnica mestiza. Con respecto a las prácticas de prevención para el CCU, se evidencia que la mayoría de participantes se realizaron más de una vez el PAP en el lapso de cinco años y antes de los 30 años de edad, cerca del $40 \%$ no se realizan oportunamente ni con la frecuencia recomendada, lo cual contrasta con los resultados del estudio efectuado en Taiwán, en el que se muestra que las mujeres de entre 20 a 49 años de edad, residentes tanto en zonas urbanas como rurales, realizaron más de 20 visitas clínicas de control y una prueba de Papanicolaou (Yang et al., 2020).

Las lesiones identificadas según clasificación Bethesda, en orden de frecuencia, en dicho grupo de mujeres están: LIEAG $21.65 \%$, LIEBG $19.69 \%$, Cáncer $14.57 \%$, ASCUS $8.66 \%$, ASC H $2.76 \%$ y ACG $1.18 \%$, que son comparables con los resultados de un estudio similar realizado en Colombia, cuyos hallazgos fueron: $19 \%$ lesiones de bajo grado, $4 \%$ de alto grado, $8 \%$ ASC-US, $2 \%$ ASC-H, 3.3\% ASG-US y 3.3\% lesión compatible con cáncer invasivo (Sousa \& Colmenares, 2019). En la presente investigación, si bien la mayoría de las mujeres con lesiones de cuello uterino poseen información sobre el VPH, menos de la mitad han afirmado conocer que el $\mathrm{VPH}$ es la principal causa del CCU, que se puede 
transmitir sexualmente y que existen vacunas para la prevención y pruebas para la detección temprana. En otro estudio realizado en Colombia se encontró un nivel de conocimiento alto respecto a la citología y la vacuna contra el virus del papiloma humano como métodos de prevención, pero el nivel fue bajo cuando se consideraron los factores de riesgo para el desarrollo de cáncer de cuello uterino (Narváez Ocampo et al., 2019).

En lo referente a las prácticas de prevención para el CCU, en la presente investigación se muestran las barreras que han dificultado la realización del examen del PAP; en primer lugar, los factores personales (falta de tiempo, no pensó que fuera importante, desconocimiento sobre cáncer cervical, descuido, no pensó que estaba en riesgo, desconocimiento sobre el PAP, miedo, no tenía molestias, vergüenza y embarazo); en segundo lugar, aquellos relacionados con el servicio de salud (desconfianza en los médicos, pérdida de exámenes por el personal de salud burocracia, incomodidad); en tercer lugar, la situación económica como la falta de dinero; y en cuarto lugar, los factores familiares (le decían que no debe hacerse el examen). En un estudio realizado en Uruguay también identificaron el miedo, la vergüenza, la incomodidad, el hecho de no estar preocupada, la falta de tiempo y la falta de conocimiento, como factores personales; en tanto que las demoras en la sala de espera y en acceder a la fecha y hora para realizarse el estudio fueron declaradas como barreras relacionadas con los servicios de salud (Rodríguez et al., 2015). En un estudio desarrollado en México las barreras detectadas fueron la religión, vivir en unión libre, antecedentes de un embarazo, nivel socioeconómico medio-bajo y medio-alto y la actitud indiferente del personal de salud (Cuevas-Martínez et al., 2016). También fueron encontrados los obstáculos de desplazamiento, temor a la prueba, desconfianza, trato poco cordial del personal de salud, entre otros (Aranguren Pulido et al., 2017).

Sin duda que el examen de Papanicolaou es de suma importancia para detectar alteraciones en las células del cuello uterino y tratar a tiempo antes que evolucione hacia un cáncer letal. En este estudio encontramos como factores que facilitaron el acceso a los servicios de salud, en orden de frecuencia, la visita de seguimiento, la recomendación del médico ya sea porque presentaba o no molestias, por iniciativa propia y por recomendación de una amiga o familiar, que es comparable con el estudio realizado en Uruguay en el cual se refiere que el principal motivo de visita al ginecólogo fue por embarazo o porque tenía algún malestar o se realizó por control (Rodríguez et al., 2015). En este marco la educación sobre derechos sexuales y reproductivos implica un asunto de poder, ya que esta incide en la conducta de las mujeres para que ejerciten sus derechos con reciprocidad y solidaridad social.

\section{CONCLUSIONES}

La mayoría de las mujeres con lesiones histopatológicas del cuello uterino atendidas en los servicios de salud poseen información acerca el VPH, de ellas una minoría conoce sobre la relación con el CCU, que se puede transmitir sexualmente, que existen vacunas para la prevención y pruebas para la detección temprana. Las mujeres que más han sido afectadas por las lesiones histopatológicas del cuello uterino están: las mayores de 50 años, con residencia en zona urbana, que no disponen de medios de transporte porque llegan a pie, aquellas que han alcanzado la educación primaria, con estado civil casada, que presentan problemas para pagar tanto las facturas como la comida y se auto identifican como mestizas.

El $40 \%$ de las participantes no se realizan el examen de PAP oportunamente ni con la frecuencia recomendada. Entre los factores facilitadores para realizarse el PAP están: la visita de seguimiento, la recomendación del médico, por iniciativa propia y por recomendación de una amiga o familiar. Se identificaron como barreras para el examen del PAP, los factores personales como falta de tiempo, no pensó que fuera importante, desconocimiento sobre cáncer cervical, descuido, no pensó que estaba en riesgo, desconocimiento sobre el PAP, miedo, no tenía molestias, vergüenza y embarazo; entre los relacionados con el servicio de salud la desconfianza en los médicos, pérdida de exámenes por el personal de salud, burocracia, incomodidad; también influyó la situación económica como la falta de dinero; y entre los factores de su entorno social, le decían que no debe hacerse el examen.

\section{REFERENCIAS}

Amengual, J., Montaño, J., Franch, P., \& Ramos, M. (2020). Supervivencia por estadio del cáncer de cuello uterino en Mallorca y factores asociados recogidos por el Registro de Cáncer. Gaceta Sanitaria, 34(6), 589594. https://doi.org/10.1016/j.gaceta.2019.04.007

Aranguren Pulido, L. V., Burbano Castro, J. H., González, J. D., Mojica Cachope, A. M., Plazas Veloza, D. J., \& Prieto Bocanegra, B. M. (2017). Barreras para la prevención y detección temprana de cáncer de cuello uterino. Investigación en Enfermería: Imagen y Desarrollo, 19(2), 129.

https://doi.org/10.11144/Javeriana.ie19-2.bpdt

Cuevas-Martínez, M. L., Vega-Pérez, M. L., TamezRodríguez, A. R., \& Castañeda-Sánchez, O. (2016). Factores de riesgo que retrasan la participación en el programa "Detección oportuna de cáncer cervicouterino". Atención Familiar, 23(4), 134-138. https://doi.org/10.1016/j.af.2016.08.003

IARC. (2007). IARC monographs on the evaluation of carcinogenic risks to humans, volume 90, Human papillomaviruses: This publication represents the views and expert opinions of an IARC Working Group on the Evaluation of Carcinogenic Risks to Humans, which met in Lyon, 15 - 22 February 2005. IARC.

Karadag Arli, S., Bakan, A. B., \& Aslan, G. (2019). Distribution of cervical and breast cancer risk factors in women and their screening behaviours. European Journal of Cancer Care, 28(2), e12960. https://doi.org/10.1111/ecc.12960

Kashyap, N., Krishnan, N., Kaur, S., \& Ghai, S. (2019). Risk Factors of Cervical Cancer: A Case-Control Study. Asia-Pacific Journal of Oncology Nursing, 6(3), 308. https://doi.org/10.4103/apjon.apjon_73_18

Krishnan, K., \& Thomas, A. (2016). Correlation of cervical cytology with high-risk HPV molecular diagnosis, genotypes, and histopathology-A four year 
study from the UAE: CERVICAL CYTOLOGY AND HPV MOLECULAR DIAGNOSIS. Diagnostic

Cytopathology, 44(2), 91-97.

https://doi.org/10.1002/dc.23391

Landy, R., Pesola, F., Castañón, A., \& Sasieni, P. (2016). Impact of cervical screening on cervical cancer mortality: Estimation using stage-specific results from a nested case-control study. British Journal of Cancer, 115(9), 1140-1146.

https://doi.org/10.1038/bjc.2016.290

Liu, Z.-C., Liu, W.-D., Liu, Y.-H., Ye, X.-H., \& Chen, S.-D. (2015). Multiple Sexual Partners as a Potential Independent Risk Factor for Cervical Cancer: A Metaanalysis of Epidemiological Studies. Asian Pacific Journal of Cancer Prevention, 16(9), 3893-3900. https://doi.org/10.7314/APJCP.2015.16.9.3893

Lorenzi, A. T., Syrjänen, K. J., \& Longatto-Filho, A. (2015). Human papillomavirus (HPV) screening and cervical cancer burden. A Brazilian perspective. Virology Journal, 12(1), 112. https://doi.org/10.1186/s12985-015-0342-0

Mijit, F., Ablimit, T., Abduxkur, G., \& Abliz, G. (2015). Distribution of human papillomavirus (HPV) genotypes detected by routine pap smear in uyghurmuslim women from Karasay Township Hotan (Xinjiang, China). Journal of Medical Virology, 87(11), 1960-1965. https://doi.org/10.1002/jmv.24240

Muñoz, N., Bosch, F. X., de Sanjosé, S., Herrero, R., Castellsagué, X., Shah, K. V., Snijders, P. J., \& Meijer, C. J. (2003). Epidemiologic classification of human papillomavirus types associated with cervical cancer. New England Journal of Medicine, 348(6), 518-527.

Muñoz, N., Franceschi, S., Bosetti, C., Moreno, V., Herrero, R., Smith, J. S., Shah, K. V., Meijer, C. J., \& Bosch, F. X. (2002). Role of parity and human papillomavirus in cervical cancer: The IARC multicentric case-control study. The Lancet, 359(9312), 1093-1101. https://doi.org/10.1016/S01406736(02)08151-5

Narváez Ocampo, L. J., Collazos Cerón, A. C., Daza Ocampo, K. T., Torres Camargo, Y. A., Ijají Piamba, J. E., Gómez Sandoval, D. M., \& Orozco Florez, C. A. (2019). Conocimientos sobre prevención y factores de riesgo para cáncer de cuello uterino en un centro de educación técnica. Revista Peruana de Ginecología y Obstetricia, 65(3), 299-304 https://doi.org/10.31403/rpgo.v66i2185

Rodríguez, G., Caviglia, C., Alonso, R., Sica, A., Segredo, S., León, I., \& Musé, I. (2015). Conocimientos, actitudes y prácticas sobre el test de Papanicolaou y estadificación del cáncer de cuello uterino. Revista Médica del Uruguay, 31(4), 231-240.

Sánchez, E. M. S. (2012). Distribución de genotipos en mujeres conizadas por lesión escamosa intraepitelial de alto grado (CIN 2-3) y análisis de los cofactores de cáncer de cérvix en Málaga [Universidad de Málaga]. http://goo.gl/5FHZlY

Schmitt, M., Depuydt, C., Benoy, I., Bogers, J., Antoine, J., Arbyn, M., Pawlita, M., \& on behalf of the VALGENT Study Group. (2013). Prevalence and viral load of 51 genital human papillomavirus types and three subtypes. International Journal of Cancer, 132(10), 2395-2403. https://doi.org/10.1002/ijc.27891

Sousa, K. D., \& Colmenares, E. (2019). Prevalencia de lesiones intraepiteliales cervicales de bajo y alto grado en pacientes en edad fértil. Maracaibo. Estado Zulia. Revista Digital de Postgrado, 8(3), e179. https://doaj.org/article/47b7efeb1da74b6d9a116b479b6 $88 \mathrm{ab} 1$

Stelzle, D., Tanaka, L. F., Lee, K. K., Ibrahim Khalil, A., Baussano, I., Shah, A. S. V., McAllister, D. A., Gottlieb, S. L., Klug, S. J., Winkler, A. S., Bray, F., Baggaley, R., Clifford, G. M., Broutet, N., \& Dalal, S. (2021). Estimates of the global burden of cervical cancer associated with HIV. The Lancet Global Health, 9(2), e161-e169. https://doi.org/10.1016/S2214109X(20)30459-9

Su, B., Qin, W., Xue, F., Wei, X., Guan, Q., Jiang, W., Wang, S., Xu, M., \& Yu, S. (2018). The relation of passive smoking with cervical cancer: A systematic review and meta-analysis. Medicine, 97(46), e13061. https://doi.org/10.1097/MD.0000000000013061

Sugawara, Y., Tsuji, I., Mizoue, T., Inoue, M., Sawada, N., Matsuo, K., Ito, H., Naito, M., Nagata, C., Kitamura, Y., Sadakane, A., Tanaka, K., Tamakoshi, A., Tsugane, S., Shimazu, T., \& Research Group for the Development and Evaluation of Cancer Prevention Strategies in Japan. (2019). Cigarette smoking and cervical cancer risk: An evaluation based on a systematic review and meta-analysis among Japanese women. Japanese Journal of Clinical Oncology, 49(1), 77-86. https://doi.org/10.1093/jjco/hyy158

Torres-Poveda, K., Ruiz-Fraga, I., Madrid-Marina, V., Chavez, M., \& Richardson, V. (2019). High risk HPV infection prevalence and associated cofactors: A population-based study in female ISSSTE beneficiaries attending the HPV screening and early detection of cervical cancer program. BMC Cancer, 19(1), 1205. https://doi.org/10.1186/s12885-019-6388-4

WHO. (2021). Temas de Salud [Estática]. Organización Mundial de la Salud. https://www.who.int/es/newsroom/fact-sheets/detail/human-papillomavirus-(hpv)and-cervical-cancer

WHO, \& IARC. (2020a). Estimated age-standardized incidence rates (World) in 2020, all cancers, both sexes, all ages. Datasource: Globocan 2020. World Health Organization \& International Agency for Research on Cancer. https://www.uicc.org/news/globocan- 2020-newglobal-cancer-data

WHO, \& IARC. (2020b). Ecuador. Source: Globocan 2020. World Health Organization \& International Agency for Research on Cancer. https://gco.iarc.fr/today/data/factsheets/populations/218 -ecuador-fact-sheets.pdf

Yang, C.-M., Sung, F.-C., Hsue, C.-S., Muo, C.-H., Wang, S.-W., \& Shieh, S.-H. (2020). Comparisons of Papanicolaou Utilization and Cervical Cancer Detection between Rural and Urban Women in Taiwan. International Journal of Environmental Research and Public Health, 18(1), 149. https://doi.org/10.3390/ijerph18010149 